\title{
Dissecção Aguda da Aorta Durante a Gravidez
}

\author{
Acute Aortic Dissection during Pregnancy \\ Walkiria Samuel Avila, Ricardo Dias, Renato Takeshi Yamada, Adriano Armelin \\ Instituto do Coração e Hospital das Clínicas da Faculdade de Medicina da USP - São Paulo, SP
}

A dissecção aguda da aorta representa uma das situações clínicas mais dramáticas na gravidez. As dificuldades do diagnóstico e a limitação da investigação invasiva elevam a taxa de mortalidade pela doença. A escassez de experiências relatadas na literatura não permite determinar uma conduta clínica e/ou cirúrgica frente à dissecção de aorta durante a gravidez. Apresentamos um caso de dissecção aguda da aorta em paciente cursando a 33a semana de gestação e discutimos sobre a conduta diante das peculiaridades da manifestação da doença.

A dissecção aguda da aorta é um evento grave e raro durante o período gestacional e representa uma das situações clínicas mais dramáticas no ciclo gravídico-puerperal. As dificuldades do diagnóstico, a valorização dos sintomas (que se confundem com os da gravidez normal) e a limitação da investigação invasiva (pelo potencial risco ao concepto) resultam na demora do tratamento e elevam, ainda mais, a taxa de mortalidade pela doença.

A heterogeneidade da apresentação dos casos e a escassez de experiências relatadas na literatura não permitem, ainda, determinar as diretrizes para a conduta clínica e/ou cirúrgica ante a dissecção de aorta durante a gravidez. Contudo, o tratamento deve sempre proporcionar chances de sobrevida à mãe e ao feto, ponderando-se o quadro clínico materno, a idade gestacional e a oportunidade da intervenção cirúrgica, quando indicada.

Apresentamos um caso de dissecção aguda da aorta em paciente cursando a $33^{\text {a }}$ semana de gestação e discutimos sobre a conduta diante das peculiaridades da manifestação da doença.

\section{Relato do Caso}

Paciente de 35 anos, médica, primigesta, nulípara, assintomática, com 33 semanas de gestação, sem antecedentes pessoais de cardiopatia ou de hipertensão arterial, apresentou forte dor retroesternal com queda da pressão arterial (80/40 $\mathrm{mmHg}$, sic), sem relação com esforço, seguida de melhora gradativa e espontânea. Foi atendida num setor de emergência médica e medicada com antiespasmódico. Evoluiu durante as 48 horas seguintes com desconforto respiratório, mal-estar e edema de membros inferiores. Nessa ocasião, foi aconselhada a realizar um ecocardiograma, o qual registrou dissecção de

\section{Palavras-chave}

Dissecção de aorta, gravidez, cirurgia cardíaca.
Acute aortic dissection is one of the most dreaded clinical conditions during pregnancy. Difficulties in establishing a diagnosis and limitations regarding invasive studies increase mortality rates associated with the disease. The limited experience reported in the literature does not allow the determination of guidelines for clinical and/or surgical management of aortic dissection in pregnancy. The authors present a case of acute aortic dissection in a woman in her $33^{\text {rd }}$ week of gestation and discuss the diagnostic approach considering the peculiarities of the disease's manifestation. aorta Tipo I, de DeBakey, insuficiência aórtica leve e importante disfunção diastólica de ventrículo esquerdo (fig. 1), sendo imediatamente encaminhada ao Instituto do Coração.

A paciente relatou que nas primeiras semanas da gestação os exames pré-natais de rotina foram normais; na $29^{a}$ semana apresentou palpitação de curta duração, o que motivou a realização de eletrocardiograma convencional e ecocardiograma transtorácico, ambos sem anormalidades; na $32^{\mathrm{a}}$ semana realizou uma urina tipo I que apresentou leucocitúria de $71.000 / \mathrm{ml}$.

Durante a internação estava consciente, dispnéica $++/++++$, acianótica, afebril e descorada $++/++++$. A pressão arterial (PA) aferida foi 130/60 mmHg e a freqüência cardíaca (FC) 90 batimentos por minuto (bpm); os pulsos periféricos eram palpáveis e simétricos, e a pressão de pulso elevada. Nos pulmões auscultava-se murmúrio vesicular

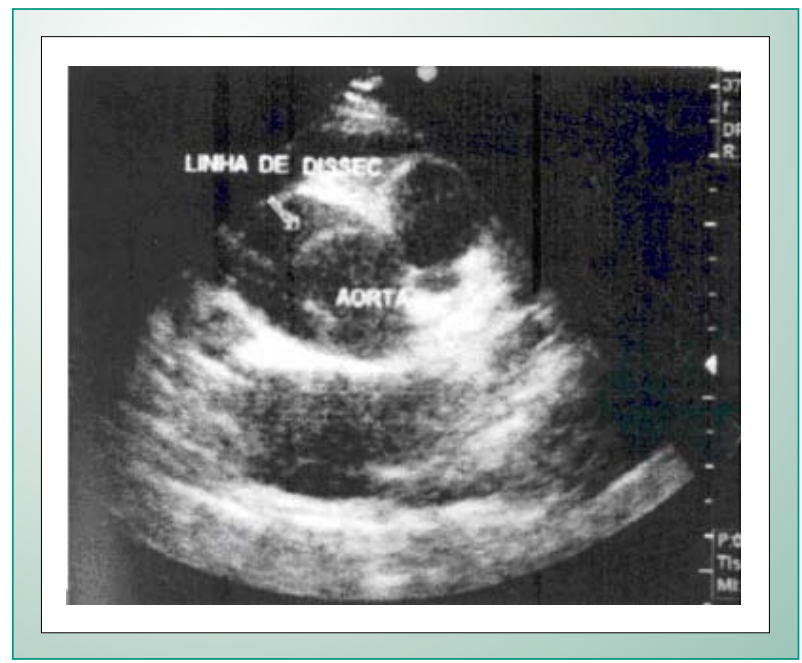

Fig. 1 - Estudo ecocardiográfico bidimensional com mapeamento colorido do fluxo da aorta que mostra a dissecção de aorta tipo 1. Realizado por Dra. Luciana Braz Peixoto em 6/10/2004, cedido pelo setor de ecocardiografia da Medicina Diagnóstica Delboni Auriemo - Diagnósticos da América. 


\section{Relato de Caso}

normal bilateralmente, sem ruídos adventícios. O coração estava rítmico, com bulhas normofonéticas e sem sopros. Os membros inferiores apresentavam edema $++/++++$; não havia hepatomegalia e o abdome evidenciava útero gravídico compatível com trinta semanas de gestação.

A tomografia, realizada na internação, mostrou diâmetros aumentados de cavidades cardíacas esquerdas e confirmou o diagnóstico de dissecção de aorta Tipo I, com acometimento do tronco braquiocefálico e da aorta ascendente e descendente (fig. 2). Os exames obstétrico e ultra-sonográfico do abdome mostraram que o feto era viável, único, tinha a vitalidade preservada, idade gestacional de 33 semanas e peso estimado em $2.300 \mathrm{~g}$.

Fundamentada no progressivo risco de ruptura da aorta e diante da viabilidade fetal, a equipe médica indicou imediata cirurgia corretiva da dissecção e interrupção da gestação por parto cesáreo antecedendo a toracotomia.

A paciente participou e concordou com a essa decisão, e entrou no centro cirúrgico consciente e com os dados vitais estáveis, como mostrou a monitorização invasiva. Quando da indução anestésica para o parto cesáreo ocorreu hipotensão súbita seguida de parada cardíaca em assistolia. Sob massagem cardíaca externa foi realizada a cesárea por laparotomia mediana e imediata retirada do bebê. Não havendo recuperação dos batimentos cardíacos maternos iniciou-se a esternotomia exploradora que evidenciou um importante hemopericárdio. Após sua drenagem, restabeleceram-se os batimentos cardíacos e se estabilizou o quadro hemodinâmico materno. A circulação extracorpórea foi estabelecida por perfusão anterógrada através do tronco braquiocefálico e drenagem venosa por meio de cânula única no átrio direito; a proteção miocárdica foi realizada com solução sangüínea fria e hipotermia a $20^{\circ} \mathrm{C}$. A correção da dissecção foi feita com interposição de tubo de dacron na aorta ascendente (fig.3) e na artéria subclávia direita, destacada que fora do tronco braquiocefálico durante a instalação da linha arterial.

O exame anatomopatológico mostrou delaminação parcial da aorta no nível da camada média e hemorragia da

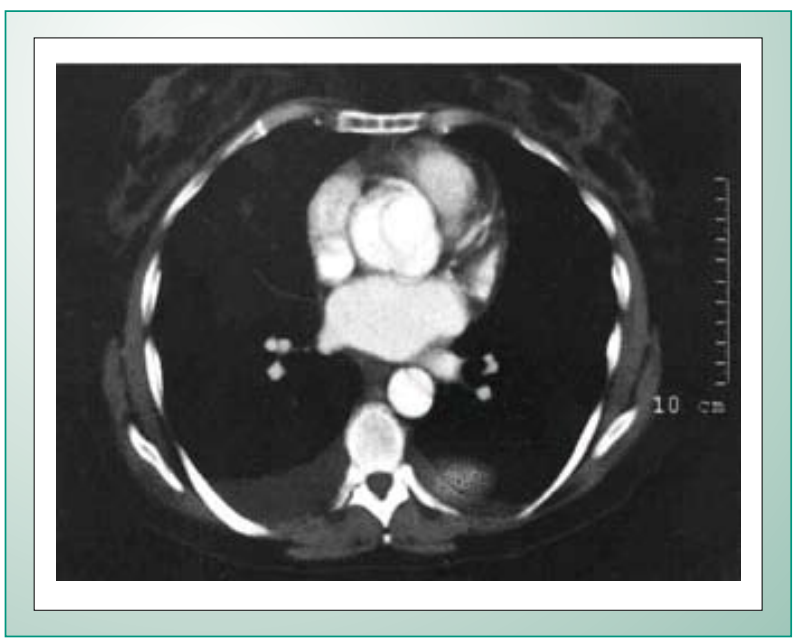

Fig. 2 - Tomografia computadorizada pré-operatória ilustrando a dissecção tipo 1. adventícia, e moderado acúmulo de substância mucóide nas camadas média e íntima.

No pós-operatório, a paciente permaneceu sob ventilação assistida durante 48 horas e foi medicada com os fármacos de rotina do pós-operatório e ocitócico em doses habituais. Manteve-se hemodinamicamente estável, sem sangramentos, e com contratilidade uterina e loquiação normais. Evoluiu nos primeiros sete dias de pós-operatório com edema ++++ de membros inferiores, hipertensão arterial importante (PA, em média, 180/120 mmHg) e taquicardia sinusal (FC, em média, $110 \mathrm{bpm})$, que foram controladas, gradativamente, com atenolol $200 \mathrm{mg} / \mathrm{dia}$, captopril $150 \mathrm{mg} / \mathrm{dia}$, amlodipina $20 \mathrm{mg} /$ dia e furosemide $80 \mathrm{mg} / \mathrm{dia}$. Administrou-se, também, heparina de baixo peso molecular, em doses profiláticas, durante seis semanas. Nos dias sucessivos as doses dos medicamentos foram ajustadas para manutenção da PA em torno de 100/60 mmHg e da FC em aproximadamente $70 \mathrm{bpm}$. A alta hospitalar ocorreu no 15으 dia de pós-operatório mantendo-se a mesma dosagem de atenolol e amlodipina e reduzindo-se a de captopril (75 $\mathrm{mg} / \mathrm{dia}$ ). Pela ausência de assepsia e antissepsia para as láparo e toracotomias de emergência, indicou-se profilaxia antibiótica com vancomicina $500 \mathrm{mg} /$ dia e ceftriaxone $1 \mathrm{~g} /$ dia durante 21 dias consecutivos.

O bebê, do sexo masculino e com idade gestacional de 33 semanas, nasceu sob anestesia geral e durante as manobras de ressuscitação cardíaca materna; apresentou bradicardia, cianose e palidez cutânea, com índices de Apgar de 1 no primeiro minuto, de 5 no quinto e de 7 no décimo minuto de vida.

Permaneceu sob ventilação artificial com oxigênio a $100 \%$ em balão anestésico no primeiro minuto, e recuperou rapidamente a freqüência cardíaca, a coloração da pele e a respiração espontânea; porém, apresentavase hipotônico e sem irritabilidade reflexa. Evoluiu com insuficiência respiratória, sendo necessárias ventilação mecânica intermitente e administração de surfactante por via endotraqueal por 2 horas, obtendo-se melhora dos parâmetros ventilatórios; foi extubado ainda no primeiro dia de vida e mantido com cateter nasal de oxigênio com pressão positiva contínua nas vias aéreas.

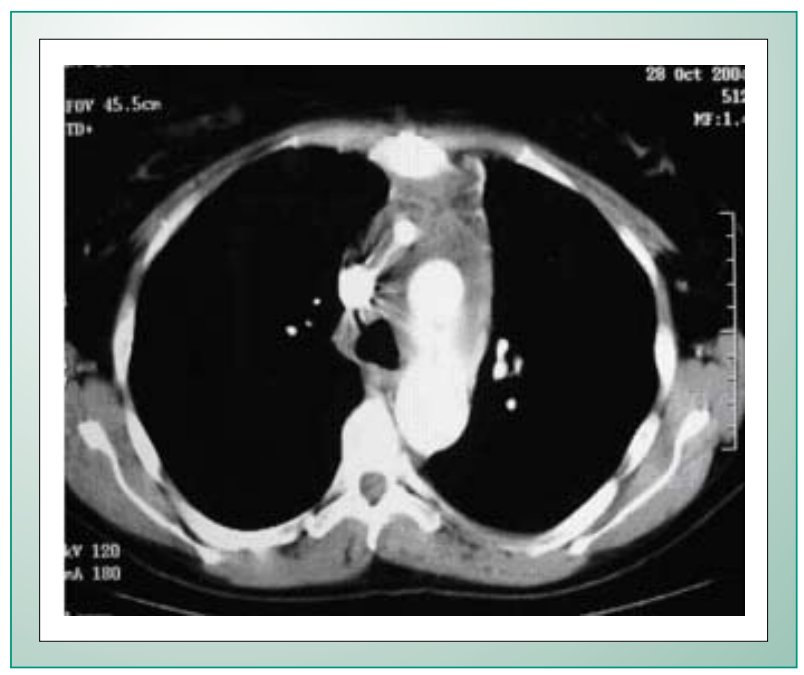

Fig. 3 - Tomografia pós-operatória ilustrando a correção da aorta ascendente com a interposição de tubo de dacron. 
No segundo dia de vida, diagnosticou-se pneumotórax à direita e saturação de oxigênio reduzida, tendo sido novamente intubado e mantido sob ventilação mecânica e agentes vasoativos. Após duas drenagens de tórax e ventilação de alta freqüência houve melhora clínica e dos parâmetros ventilatórios, o que lhe permitiu ser extubado em seu nono dia de vida, embora continuasse na incubadora e com cateter de oxigênio. Com a melhora do quadro respiratório foi diminuída, progressivamente, a administração de oxigênio e de fármacos vasoativos. Os drenos torácicos foram retirados em seu $12^{\circ}$ dia, ocasião em que se iniciou a alimentação enteral, através de sonda orogástrica.

Com dificuldade de coordenação na sucção-deglutição, foi necessária estimulação não-nutritiva e transição paulatina para a alimentação oral. Embora apresentasse hemograma normal e hemocultura negativa, a criança foi mantida sob antibioticoterapia durante dez dias, porque o exame radiológico mostrava velamento na base pulmonar direita, a proteína C-reativa apresentava-se elevada para a idade $(6,8)$ e havia história materna de infecção urinária na ocasião do parto. O exame neurológico foi normal, assim como o ecocardiograma Doppler e a ultra-sonografia cerebral; porém, a tomografia cerebral evidenciou atenuação de grau leve da substância branca em ambos os hemisférios cerebrais. Respondendo adequadamente aos estímulos de sucção e recebendo todo volume de leite por via oral, o bebê teve alta hospitalar com 34 dias de vida, em boas condições.

\section{Discussão}

A dissecção de aorta na mulher jovem é uma doença incomum, de etiologia às vezes desconhecida, potencialmente catastrófica e de extensão variável, que se inicia com a ruptura da íntima dessa artéria.

Em pacientes jovens, as condições clínicas mais comuns que predispõem à dissecção de aorta são as doenças do colágeno (Marfan, Ehlers-Danlos) ou aquelas que primariamente acometem o vaso, como a coarctação e a valva aórtica bivalvular ${ }^{1}$. Contudo, em mulheres saudáveis tem sido descrita uma forte correlação de dissecção da aorta e gravidez, fundamentada na alta incidência desse evento durante a gestação ${ }^{2,3}$. De fato, a revisão de Schnitker e Bayer verificou que 24 das 49 delaminações aórticas em mulheres abaixo de quarenta anos de idade ocorreram em associação com a gravidez, e vinte delas aconteceram antes do parto ${ }^{4}$. A causa dessa associação (gravidez/dissecção de aorta) em mulheres internadas como saudáveis ainda não está claramente estabelecida.

Inicialmente sugeriu-se que incrementos fisiológicos da volemia e do débito cardíaco poderiam ser as potenciais causas do evento; contudo, essa hipótese não consegue explicar a menor incidência de dissecção durante o trabalho de parto ${ }^{5}$, ocasião em que, em relação a outras fases do ciclo gravídico-puerperal, acontece o maior impacto circulatório em razão da importante oscilação hemodinâmica.

Tem-se cogitado, também, que as modificações estruturais vasculares demonstradas durante a gravidez, semelhantes à necrose cística da média, responsáveis pelo aumento do diâmetro e da complacência da aorta (mais marcantes no termo da gestação), possam desempenhar um papel importante na dissecção ${ }^{5}$. A degeneração mucóide nas camadas média e íntima, descrita no exame anatomopatológico da aorta do presente caso, coincide com os relatos da literatura sobre as dissecções que ocorrem no terceiro trimestre da gravidez. Nesse aspecto, destaca-se o recente estudo genético que demonstrou expressão variável de genes de transcrição das enzimas metaloproteinases da matriz celular da aorta que apresentam forte influência na remodelação vascular fisiológica da gravidez $z^{6}$.

Com respeito aos hormônios, ainda é desconhecida sua exata influência sobre o tecido conectivo vascular. Estudos em modelos animais, contudo, demonstraram que os estrógenos inibem o colágeno e a deposição de elastina na aorta enquanto a progesterona acelera, minimamente, a deposição de proteínas não-colagenosas nas paredes do vaso ${ }^{7}$.

As manifestações clínicas da dissecção aórtica, expressas classicamente por forte dor retroesternal e/ou abdominal, foram, nesse caso, subestimadas e indiferenciadas da dor torácica decorrente dos efeitos mecânicos do útero gravídico e da obstipação intestinal, comuns na gravidez normal. A propósito, ainda, do diagnóstico diferencial, devem ser sempre considerados a embolia amniótica, o descolamento prematuro da placenta, a ruptura uterina e o pneumotórax espontâneo como causas freqüentes de dor torácica durante a gravidez.

Embora apresente limitações para o diagnóstico de dissecção da aorta, o ecocardiograma mostrou-se útil na investigação inicial, por ser um exame não-invasivo e de fácil execução. A tomografia computadorizada, com as vantagens da rápida factibilidade e do relativo baixo custo, confirmou o diagnóstico e, principalmente, a classificação da dissecção, dado esse de fundamental importância para definir a conduta terapêutica.

A localização da delaminação na aorta ascendente com extensão para a descendente caracterizou o presente caso como Tipo I, de DeBakey, o que implicou correção cirúrgica imediata, uma vez que o tratamento clínico desse tipo de dissecção associa-se a progressivas taxas de mortalidade, alcançando $80 \%$ de óbito na primeira semana após o diagnóstico ${ }^{8}$.

Nos últimos anos têm-se observado três dados relevantes para levar a bom termo o tratamento dessa grave afecção: o encaminhamento precoce desses pacientes para a cirurgia, a maior experiência do cirurgião e a evolução dos cuidados no pós-operatório. Mesmo assim, a cirurgia corretiva de dissecção aórtica ainda se associa a graves complicações cardíacas e taxas de mortalidade cirúrgica estimadas entre $9 \%$ e $36 \%{ }^{9}$. Não diferente do risco materno, a cirurgia cardíaca impõe ao feto riscos de morbidade e de mortalidade de aproximadamente $30 \%$ e $9 \%$, respectivamente, porcentuais variáveis de acordo com o tipo de procedimento, tempo de circulação extracorpórea, hipotermia, fluxo não-pulsátil e condições clínicas prévias maternas ${ }^{10}$.

No presente caso, a indicação prioritária de interrupção da gravidez, anterior à correção da dissecção, teve por finalidade preservar ambas as vidas, a da mãe e a do concepto, uma vez que esse, já viável, teria, assim, maiores chances de sobrevivência do que se fosse mantido intra-útero durante essa cirurgia de alta complexidade. Ponderou-se, para assunção dessa conduta, o elevado risco de possível sofrimento fetal agudo, o que implicaria cesárea de emergência durante o 


\section{Relato de Caso}

intra ou o pós-operatório materno, agravando seriamente o prognóstico da paciente.

Nossa conduta esteve de acordo com os dados descritos na literatura os quais baseiam os melhores índices de sobrevida (mãe e feto) na idade gestacional. Assim, se o evento ocorrer até a 28a semana da gestação, a cirurgia para correção da delaminação aórtica deve ser realizada com o feto intraútero; após 32 semanas, condição em que o feto está viável, a intervenção corretiva deve ser feita depois da cesárea. Menos pacífica é a conduta a ser tomada se a dissecção aórtica ocorrer no período entre 28 e 32 semanas, quando o parto deve ser definido pelas condições do concepto, indicando-se a cesárea se houver sofrimento fetal ou mantendo-se a gravidez se o feto tolerar a cirurgia materna ${ }^{11}$.

O tamponamento cardíaco e a subseqüente parada cardíaca alertam, energicamente, para o risco e a gravidade da demora em se realizar o procedimento cirúrgico. No presente caso, a laparotomia, antecedendo a toracotomia, resgatou o concepto e liberou o cirurgião para reparar a aorta sem a preocupação adicional do feto intra-útero. O sucesso obtido deveu-se, sem dúvida, à indicação da intervenção cirúrgica na aorta imediatamente depois de confirmado o diagnóstico.

As condições clínicas apresentadas pelo bebê ao nascimento pressupõem sofrimento intra-uterino agudo. A resposta satisfatória às manobras iniciais de reanimação permite supor que essa asfixia foi de curta duração; entretanto, foi suficiente para comprometer, ainda que transitoriamente, o sistema nervoso central, demonstrado pela demora na recuperação do tônus e da irritabilidade reflexa. Embora o exame ultra-sonográfico tenha sido normal, espera-se, nos prematuros com asfixia perinatal importante, a ocorrência de hemorragia intracraniana ${ }^{12,13}$.

Na idade gestacional de 33 semanas a produção de

\section{Referências}

1. Spittell PC, Spittell JA, Joyce JW, et al. Clinical features and differential diagnosis of aortic dissection: experience with 236 cases (1980 through 1990). Mayo Clin Proc 1993; 68: 642-51.

2. Barrett JM, Van Hooydonk JE, Boehm FH. Pregnancy related rupture of arterial aneurysms. Obstetr Gynecol Survey 1982; 37: 557-66.

3. Oskoui R, Lindsay J Jr. Aortic dissection in women $<40$ years of age and the unimportance of pregnancy. Am J Cardiol 1994; 73: 821-3.

4. Schnitker MA, Bayer CA. Dissecting aneurysm of the aorta in young individuals, particularly in association with pregnancy. With report of a case Ann Inter Med 1944; 20: 486-511.

5. Anderson RA, Fineron PW. Aortic dissection in pregnancy: importance of pregnancy-induced changes in the vessel wall and bicuspid aortic valve in pathogenesis. Br J Obstet Gynaecol 1994; 101: 1085-8.

6. Kelly BA, Bond BC, Poston L. Aortic adaptation to pregnancy: elevated expression of matrix metalloproteinases- 2 and -3 in rat gestation. Mol Hum Reprod 2004; 10: 331-7.

7. Wolinsky H. Effects of estrogen and progestogen treatment on the response of the aorta of male rats to hypertension. Circ Res 1972; 30: 341-9.

8. Masuda Y, Yamada Z, Morooka N, Watanabe S, Inagaki Y. Prognosis of surfactante é ainda insuficiente para manter a adequada distensão alveolar fetal. Essa situação é agravada pela asfixia, que favorece maior inativação e aumento do consumo do surfactante. Por isso seu uso tem sido indicado para melhorar o quadro respiratório, favorecendo a retirada mais precoce da ventilação mecânica ${ }^{13}$.

Os traumas pulmonares decorrentes de variações de pressão e de volume estão mais associados ao uso de surfactante sob ventilação mecânica, enquanto o pneumotórax é menos comum em pacientes sob pressão alveolar positiva. Apesar disso, a re-expansão total dos pulmões foi obtida pela drenagem bilateral e não houve seqüelas pulmonares.

O déficit de sucção-deglutição depois da 34aㅡ semana de gestação pode sinalizar algum grau de comprometimento do sistema nervoso central ${ }^{14}$. Contudo, a criança respondeu bem ao estímulo da sucção e a avaliação neurológica foi considerada normal para a idade, resultando em bom prognóstico.

Após a alta hospitalar é obrigatória a orientação a respeito do planejamento familiar, considerando-se os altos riscos de nova gravidez em pacientes que apresentam doenças de aorta. As modificações vasculares não se normalizam após o término da gestação o que implica predisposição a novos eventos cardiovasculares. Por esse motivo, é consenso considerar que as doenças da aorta apresentam contra-indicação à gravide $z^{15}$, sendo a esterilização pela laqueadura tubárea a contracepção mais efetiva para esses casos.

\section{Agradecimentos}

Ao Dr. Leon Gustavo dos Reis Macedo, médico-residente do Instituto do Coração que acompanhou a paciente no período do pós-operatório, e à Dra. Maéve Barros Correia, médica hemodinamicista, pela revisão ortográfica do texto.

patients with medically treated aortic dissections. Circulation 1991; 84: 111-7.

9. David TE, Armstrong S, Ivanov J, Barnard S. Surgery for acute type A dissection. Ann Thorac Surg 1999; 67: 1999-2001.

10. Weiss BM, Von Segesser LK, Alon E, Seifert B, Turina MI. Outcome of cardiovascular surgery and pregnancy. A systematic review of the period 1984-1996. Am J Obstet Gynecol 1998; 179: 1643-53.

11. Zeebregts CJ, Schepens MA, Hameeteman TM, et al. Acute aortic Dissection Complicating Pregnancy. Ann Thorac Surg 1997; 64: 1345-8.

12. Mancini MC, Barbosa NE, Banwart D, et al. Intraventricular hemorrhage in very low birth weight infants: associated risk factors and outcome in the neonatal period. Rev Hosp Clín Med S Paulo 1999; 54: 151-4.

13. Goldsmith LS. Immediate improvement in lung volume after exogenous surfactant: alveolar recruitment versus increased distention. J Pediatr 1991; 119: 424

14. Neiva FCB, Leone CR. Sucking development in preterm newborns and the influence of nonnutritive sucking stimulation. Pediatr Res 2003; 53: 498.

15. Diretrizes sobre cardiopatia e gravidez e planejamento familiar da mulher cardiopata. Arq Bras Cardiol 1999; 72 (supl III): 6. 